Article

\title{
Genome-Wide Identification and Characterization of the TCP Gene Family in Cucumber (Cucumis sativus L.) and Their Transcriptional Responses to Different Treatments
}

\author{
Haifan Wen ${ }^{1}$, Yue Chen ${ }^{1}$, Hui Du ${ }^{1}$, Leyu Zhang ${ }^{1}$, Keyan Zhang ${ }^{1}$, Huanle He ${ }^{1}$, Junsong Pan ${ }^{1}$, \\ Run Cai ${ }^{1,2}$ and Gang Wang ${ }^{1, *}$ \\ 1 School of Agriculture and Biology, Shanghai Jiao Tong University, Shanghai 200240, China; \\ haifanwen@sjtu.edu.cn (H.W.); yuechen321@sjtu.edu.cn (Y.C.); duhui1122@sjtu.edu.cn (H.D.); \\ zly_sjtu@sjtu.edu.cn (L.Z.); lnykzky2016@sjtu.edu.cn (K.Z.); hlhe75@sjtu.edu.cn (H.H.); \\ jspan71@sjtu.edu.cn (J.P.); cairun@sjtu.edu.cn (R.C.) \\ 2 State Key Laboratory of Vegetable Germplasm Innovation, Tianjin 300384, China \\ * Correspondence: wg770801@sjtu.edu.cn
}

Received: 16 October 2020; Accepted: 16 November 2020; Published: 20 November 2020

\begin{abstract}
TCP proteins are plant-specific transcription factors widely implicated in leaf morphogenesis and senescence, flowering, lateral branching, hormone crosstalk, and stress responses. However, the relationship between the transcription pattern of TCPs and organ development in cucumber has not been systematically studied. In this study, we performed a genome-wide identification of putative TCP genes and analyzed their chromosomal location, gene structure, conserved motif, and transcript expression. A total of 27 putative TCP genes were identified and characterized in cucumber. All 27 putative CsTCP genes were classified into class I and class II. Class I comprised 12 CsTCPs and Class II contained 15 CsTCPs. The 27 putative CsTCP genes were randomly distributed in five of seven chromosomes in cucumber. Four putative CsTCP genes were found to contain putative miR319 target sites. Quantitative RT-PCR revealed that 27 putative CsTCP genes exhibited different expression patterns in cucumber tissues and floral organ development. Transcript expression and phenotype analysis showed that the putative CsTCP genes responded to temperature and photoperiod and were induced by gibberellin (GA)and ethylene treatment, which suggested that CsTCP genes may regulate the lateral branching by involving in multiple signal pathways. These results lay the foundation for studying the function of cucumber TCP genes in the future.
\end{abstract}

Keywords: cucumber; expression pattern; TCP genes; organ development

\section{Introduction}

Transcription factors play significant roles in plant growth development. The TCP gene family is a plant-specific transcription factor family [1]. The name of TCP originated from the first four characterized members: TB1 (TEOSINTE BRANCHED 1) in Zea mays [2], CYC (CYCLOIDEA) in Antirrhinum majus [3], and the PCF1 and PCF2 (PROLIFERATING CELL FACTORS 1 and 2) in Oryza sativa [4]. The TCP transcription factors contain a conserved TCP domain, which constitutes a basic helix-loop-helix (bHLH) structure at the N-terminus. This domain is important for DNA binding and involved in protein-protein interactions [5].

According to the amino acid sequence similarity of the TCP domain, TCP factors were divided into two major classes: class I (PCF or TCP-P class) and class II (TCP-C class) [5,6]. The most significant difference between these two classes is a four-amino acid deletion in the basic domain of class I relative to class II proteins. The class II group can be further divided into subclasses, CIN and 
CYC/TB1, mainly based on differences in amino acid sequence, especially in the basic region of the TCP domain. Most members of the CYC/TB1 subclass containing an 18-20-residue arginine-rich motif ( $\mathrm{R}$ domain), but little members of the CIN subclass contain the R domain [6-8]. Up to now, many TCP genes have been confirmed in various plant species. In silico studies on TCPs have identified 24 TCPs in Arabidopsis, 28 TCPs in O. sativa [8-10], 38 TCPs in Gossypium raimondii [11], and 23 TCPs in Phalaenopsis equestris [12]. TCP and their homologs are regulated by the conserved microRNA miR319 in different species. The miR319-regulated TCPs are involved in regulating the leaf morphogenesis [13], flower architecture [14], hormone biosynthesis and response [15]. In Arabidopsis, AtTCP2-4, AtTCP10, and AtTCP24 are belong to the CIN family members which contain miR319 binding site.

Recent studies have shown that TCPs play crucial roles in plant growth and development. Several TCPS are implicated in trichome formation [16], gametophyte development [17], seed germination [18], floral development [19], and lateral shoot initiation [20]. Moreover, TCPs are also regulated by multiple hormone and external environmental signals. GhTCP14 is a crucial regulator in auxin-mediated elongation of cotton fiber cells [16]. In Arabidopsis, TCP15 is induced by gibberellins (GAs) and TCP1 is a positive regulator of the brassinosteroid (BR) biosynthesis pathway [21,22]. Besides, TCPS also participate in ethylene, strigolactone (SL) and cytokinins (CK) biosynthesis [20,23]. TCP20 and TCP22 have a positive effect on regulating the circadian clock, and TCP15 is induced by elevated temperature in Arabidopsis [24].

Cucumber (Cucumis sativus L.) is one of the most important vegetable crops worldwide [25]. Because of the diversity of flower sexual types, cucumber has become a model plant for researching the sex differentiation mechanism [26]. Despite the function of TCP genes are important, only a few cucumber TCPS were reported. TEN(CsTCP21), a TCP from cucumber, regulates the identity of tendrils $[27,28]$. CsBRC1(CsTCP3) inhibits lateral bud outgrowth by controlling auxin accumulation in axillary buds in cucumber [29]. Based on the first version of the cucumber genome, Yuan et al. identified 22 TCPs and performed bioinformatics analysis on them [30].

In this study, according to the second version of the cucumber genome with more detailed annotations, we identified 27 putative TCP genes and analyzed their chromosomal distribution, gene structure, conserved motif, phylogenetic relationship, and cis-acting elements on the promotors. We further analyzed TCPs expression profiles in different organs and response to the hormone treatment and environmental stresses in cucumber. The results should lay the foundation for studying the function of CSTCPs in the future.

\section{Materials and Methods}

\subsection{Plant Materials, Growth Conditions, and Treatment}

The cucumber monecious line 9930 was used to analyze gene expression patterns. Seeds were germinated on a wet filter paper in a Petri dish at $28^{\circ} \mathrm{C}$ in the dark overnight. The seedings were then grown in a growth room of Shanghai Jiao Tong University (Shanghai, China) for $16 \mathrm{~h} / 8 \mathrm{~h}$ at $25^{\circ} \mathrm{C} / 18^{\circ} \mathrm{C}$ (day/night). At the two true leaf stage, the cucumber seedlings were used for exogenous hormone treatment. The apical shoots of seedings were soaked in water containing $100 \mu \mathrm{M}$ gibberellin (GA) and $10 \mu \mathrm{M}$ ethylene with $0.1 \%(v / v)$ Tween-20 for $2 \mathrm{~h}$, respectively. The treatment with water containing $0.1 \%(v / v)$ Tween-20 was the control. After soaking, the apical shoots of seedings were sampled at 12 and $24 \mathrm{~h}$, respectively. Some treated seedings were used for phenotype observation.

From sowing to the fourth true leaf unfolding, some seedings were grown in plant growth chambers under four different treatments, including low temperature $\left(23^{\circ} \mathrm{C} / 15^{\circ} \mathrm{C}\right.$, day/night) and short day $(8 \mathrm{~h} / 16 \mathrm{~h}$ day/night) (LS), low temperature and long day ( $16 \mathrm{~h} / 8 \mathrm{~h}$ day/night) (LL), high temperature $\left(32{ }^{\circ} \mathrm{C} / 24^{\circ} \mathrm{C}\right.$, day/night) and short day (HS), and high temperature and long day (HL). The treatment under high temperature with a photoperiod of $16 \mathrm{~h} / 8 \mathrm{~h}$ (day/night) (HL) was a normal condition, considered to be the control. In addition, all treatments were repeated at least three times, and there were at least 15 seedings for each treatment. All materials were harvested and frozen immediately in liquid nitrogen and kept at $-80^{\circ} \mathrm{C}$ for RNA isolation. 


\subsection{Identification of TCP Genes in Cucumber}

The whole sequence data of cucumber were obtained from the Cucurbit Genomics Database (CuGenDB; ftp://cucurbitgenomics.org/pub/cucurbit/genome/cucumber/Chinese_long/v2/). The TCP sequences of 24 Arabidopsis TCPs were retrieved from the Arabidopsis Information Resource (TAIR; https: //www.arabidopsis.org/index.jsp). Two-step BLAST method was adopted to identify cucumber TCP genes. First, Arabidopsis TCPs were used to search possible cucumber TCPs with TBtools (e-value, 1e-5) [31]. Subsequently, all possible cucumber TCPs were further identified using National Center for Biotechnology Information (NCBI; https://www.ncbi.nlm.nih.gov/) BLASTP (e-value, 1e-5). Finally, candidate proteins were confirmed with SMART (http://smart.embl.de/) [32] and Pfam databases (http://pfam.xfam.org/) [33].

\subsection{Phylogenetic Tree and Cis-Acting Elements Analysis}

TCP protein sequences of 27 CsTCPs and 24 AtTCPs were used for phylogenetic analysis. The phylogenetic tree was constructed through the neighbor-joining (NJ) method using MEGAX software v.10.1.8 (https://www.megasoftware.net/) and the bootstraps test was carried out with 1000 iterations. The results were formatted for display using the Evolview V3 (https://www.evolgenius. info//evolview/\#login) [34]. The gene structure of cucumber TCP genes was identified via TBtools. The online Multiple Expectation Maximization for Motif Elicitation (MEME) program version 5.0.5 (http://meme-suite.org/tools/meme) was used to predicted conserved motifs of the cucumber TCPs [35]. Finally, 2000 bp sequences upstream of the start codon ATG of each TCP gene was used to analyze the cis-acting elements using PlantCARE (http://bioinformatics.psb.ugent.be/webtools/plantcare/html/).

\subsection{Analysis of Chromosomal Location and Collinearity Relationship and Prediction of miR319 Target Genes}

The distribution information of cucumber TCP genes on the chromosomes was acquired using TBtools. Gene Collinearity analysis of TCP genes in different species was performed using the Multiple Collinearity Scan toolkit (MCScanX) with default parameters [36]. We plotted the collinearity relationship of the TCP genes from selected species using the TBtools software. Finally, psRNATarget (http://plantgrn.noble.org/psRNATarget/) was used to identify miR319 target genes by analyzing the full length of 27 CsTCPs.

\subsection{Transcript Expression Analysis of TCP Genes}

To analyze the TCP genes expression in different organs of cucumber, we sampled 11 organs of cucumber: root (3 week old seedings), stems (12 week old cucumber plants), leaf (3 week old seedings), cotyledon ( 3 week old seedings), tendril (12 week old cucumber plants), petal, stamen (1 day before flowering), pistil (1 day before flowering), carpel (1 day before flowering), pericarp (1 day before flowering) and trichome (1 day before flowering). In addition, the expression of 27 CsTCPS in flower buds at different development stages were also examined using real-time quantitative reverse transcription PCR (real-time qRT-PCR). The gene expression pattern analysis in different flower bud development periods was taken from the buds of different lengths, 1, 2, 5 and $10 \mathrm{~mm}$.

Total RNA was extracted using OminiPlant RNA Kit (CWBIO, Beijing, China). The first-strand cDNA was synthesized using HiFiScript cDNA Synthesis Kit (CWBIO). Real-time qRT-PCR with UltraSYBR Mixture (CWBIO) was performed according to the manufacturers' protocol. CsActin3 (Csa6G484600.1) was used as the internal control. All the qRT-PCR primers were designed using the Genious software according to the cDNA sequences (Tables S1 and S2). The $2^{-\Delta \Delta C t}$ method was used to analyze the relative mRNA expression [37]. Each expression was repeated for three times biologically and technically under identical conditions. 


\section{Results}

\subsection{Identification and Chromosomal Distributions Analysis of Putative TCP Genes in Cucumber}

We used a two-step BLAST method to identify CsTCPS from cucumber genome using the TCPS sequences of Arabidopsis. In total, 27 candidate CsTCPs were screened out, all of which were verified the existence of the TCP domains with the SMART and Pfam databases. The SMART analysis revealed that all putative cucumber TCP proteins contained the TCP domain, implying that there are at least 27 putative TCP genes in cucumber. The 27 putative TCPS were named CsTCP1 to CsTCP27 according to their accession number. These TCPs varied in their coding sequence (CDS), amino acid sequence, isoelectric point (pI), and molecular weight (MW) (Table S2). From the 27 CsTCPs, CsTCP18 is the smallest protein with 174 amino acids, whereas CsTCP27 is the largest protein with 449 amino acids. The protein MWs ranged from $18.91 \mathrm{kDa}$ (CsTCP18) to $164.89 \mathrm{kDa}$ (CsTCP27), and the pI value varied from 5.57 (CsTCP24) to 9.92 (CsTCP15). The 27 putative CsTCP genes are distributed on chromosome (Chr) 1, 3, 4, 5 and 7 (Figure S1). Among them, Chr1 contained the highest number of putative TCP genes, while no putative TCP genes were found on Chr2 and Chr7.

\subsection{Phylogenetic Analysis and Classification Putative TCP Genes in Cucumber}

We constructed an unrooted phylogenetic tree using the neighbor-joining method (Figure S2, Figure 1a). The 51 TCPs from Arabidopsis and cucumber were divided into two classes, Class I (blue) and Class II, Class II could be further classified into two subclasses, CYC/TB1 and CIN. All Arabidopsis TCPs fell in the same Class as previously reported (Figure S2) [9]. There were 12 CsTCPs in class I. In the CYC/TB1 and CIN subgroup, there were six and nine CsTCPs, respectively (Figure S2, Figure 1a).
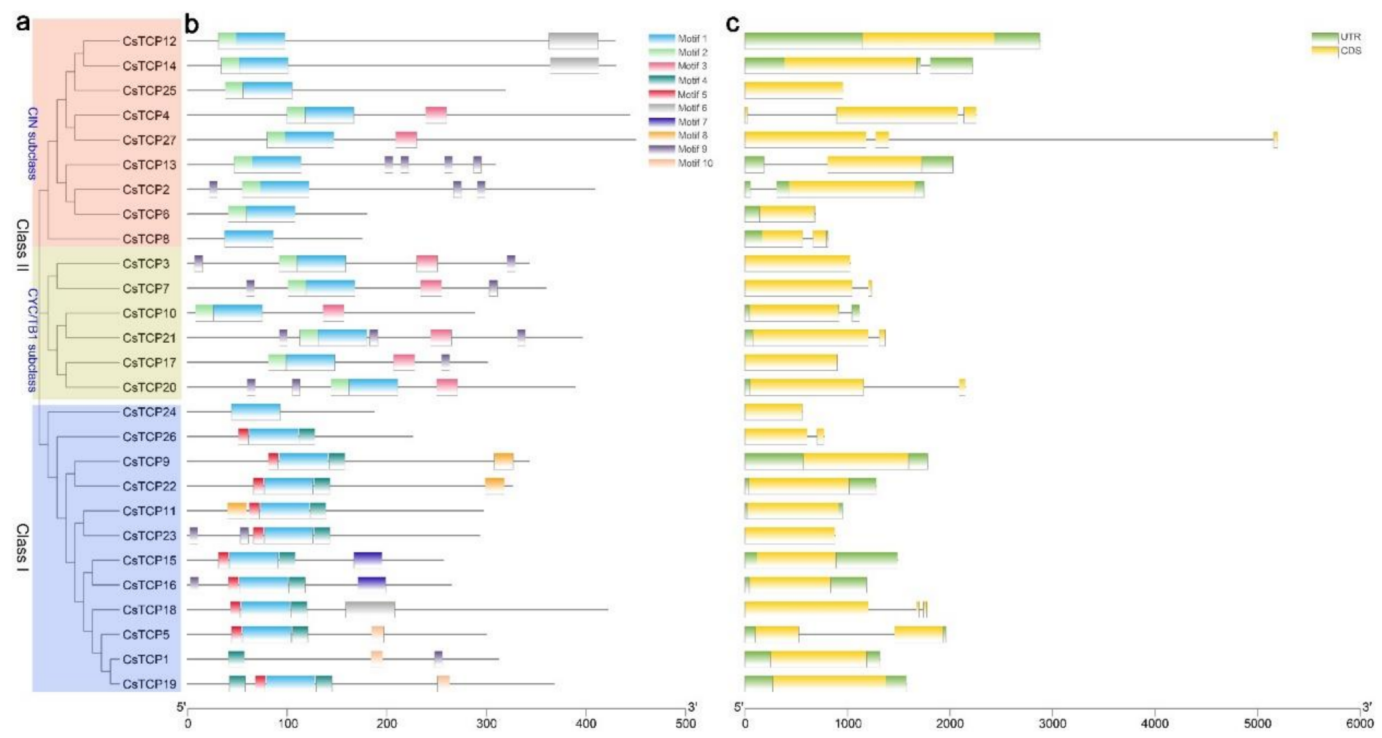

Figure 1. Motif composition and gene structure of cucumber putative TCP genes. (a). Phylogenetic tree of 27 cucumber TCPs. A specific color indicates each of the three TCP classes. (b). Motif composition of TCPs. Conserved motifs in the CsTCPs are shown in different colored boxes. (c). Gene structure analysis of putative TCP genes. Coding sequences (CDS), untranslated region (UTRs) and introns are represented by yellow boxes, green boxes, and black lines, respectively.

\subsection{TCP Gene Structures and Conserved Motifs}

According to the genome sequences and corresponding coding sequences of TCPs in cucumber, we found that the genome sequence lengths of CsTCP ranged from 561 to 5195 bp, and the lengths of CDS ranged from 525 to $1350 \mathrm{bp}$. The number of introns of these genes varies from zero to two. 
Two-thirds of CSTCPS contain only one exon and the other genes contain two or three exons, which is similar to the structure of the TCP family in other species genomes $[11,38,39]$.

Ten conserved motifs labeled Motif 1 to Motif 10 were found in CsTCPs (Figure 1b). Motif 1 was annotated as the conserved bHLH structure, and presented in almost CsTCPs except CsTCP1, suggesting that it might be necessary for the CsTCPs to serve their function. Motifs 4, 7, 8 and 10 were only presented in Class I, while Motifs 2 and 3 were only presented in Class II. Thus, CsTCPs in the same class had similar motif composition while divergence was found in two class, indicated that CsTCPs in same class may perform the similar function and that some of motifs may play a vital role in specific function.

\subsection{Collinearity Analysis of the Relationship among Cucumber, Melon (Cucumis melon) and Arabidopsis Members}

Combined analysis of TCPs from cucumber, melon and Arabidopsis was performed to study the gene collinear relationship among them (Figure 2). Collinearity analysis indicated that only seven $(25.9 \%)$ collinear gene pairs within cucumber and Arabidopsis genomes. There was no gene on Arabidopsis Chr4 that was collinear with any putative TCP genes in cucumber genome. A total of $24(88.9 \%)$ pairs of putative TCP genes were collinear between cucumber and melon (Table S3). These results indicated that putative TCP genes in cucumber and melon are highly conservative in evolution.

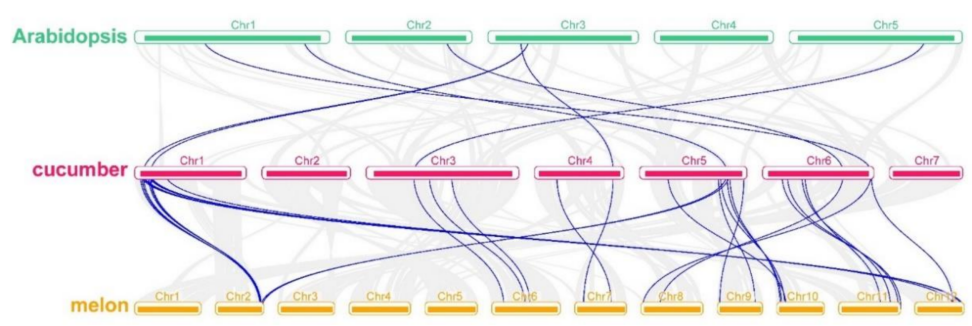

Figure 2. Collinear relationships of genes pairs from cucumber, melon and Arabidopsis. Blue lines indicate the collinear TCP gene pairs.

Based on the genome sequence analysis between cucumber and melon [40], cucumber Chr 1, 2, 3, 5 and 6 were collinear with melon Chr 2 and 12, 3 and 5, 4 and 6,9 and 10, and 8 and 11, respectively. A few putative TCP genes does not conform to this correspondence. For example, cucumber Csa5G605000 (Chr5) and Csa5G608320.1 (Chr5) were collinear to melon MELO3C017168.2.1 (Chr2) and MELO3C017286.2.1 (Chr2), respectively. Csa6G524000.1 (Chr6) was collinear to MELO3C007121.2.1 on Chr 11 and MELO3C002754.2.1 on Chr12 simultaneously. These results implied that the function of these genes may have diverged in cucumber and melon during evolution.

In cucumber, the closest evolutionary TCPs with these genes are CsTCP27, CsTCP25, CsTCP14 and CsTCP12, which contain sequences well matched with miR139 in the coding regions and might be the targets of miR319 (Figure 3). The other CsTCPs did not contain putative miR319 recognition site. These results indicated that the miR319 target sequence were conserved during the evolution of plants.

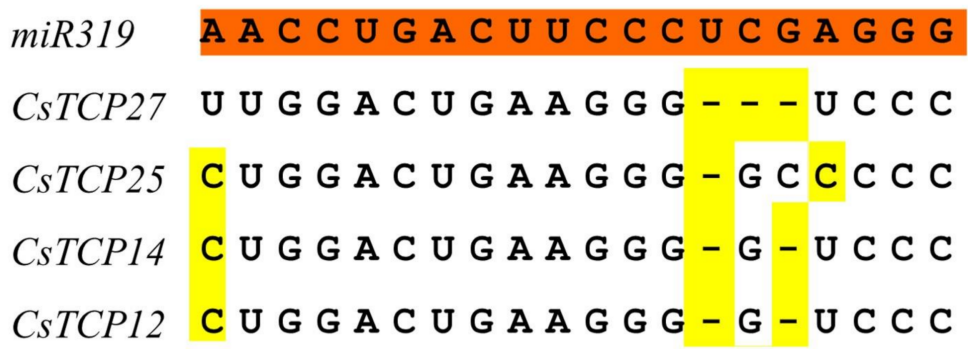

Figure 3. Alignment of putative target areas for miR319. Mismatches were represented by yellow. 


\subsection{Promotor Cis-Acting Element Analysis of Putative CsTCP Genes}

The cis-acting elements in the promoter of gene usually regulate gene expression pattern and location. In this study, a series of cis-acting elements involved in hormone response elements, light response elements, defense and stress response elements, low temperature wound response, and meristem expression were identified in putative CsTCP genes promotors (Figure 4 and Table S4).

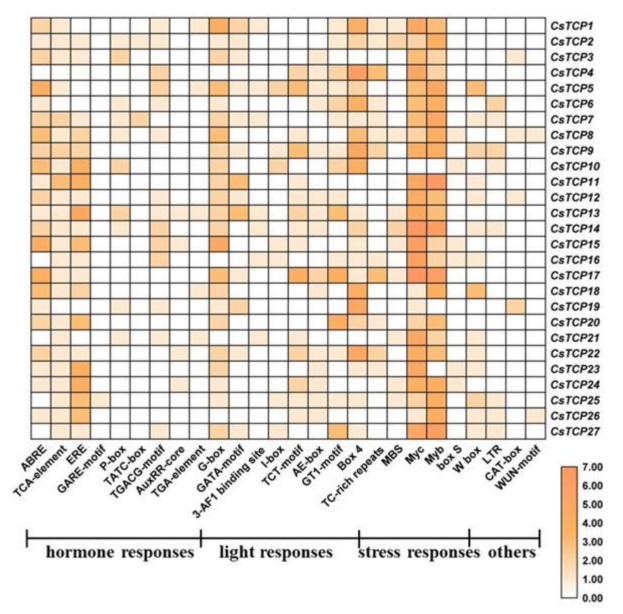

Figure 4. Cis-acting elements on promotors of CsTCPs. The color bar shows the number of cis-acting elements.

For hormone-related cis-acting elements, we found abscisic acid (ABA) response elements (ABREs) and at least two or more ABRE cis-acting elements in the CsTCPs promotors, except CsTCP4, CsTCP16, CsTCP25, CsTCP26 and CsTCP27. The salicylic acid (SA) response elements (TCA element) are relatively more extensively distributed, except CsTCP4, CsTCP6 and CsTCP19. The ethylene response element (ERE) were found in three-quarters of these promotors. We also identified other hormone-related cis-acting elements, such as auxin response elements AuxRP and TGA elements, GA response elements GAREs, P-box and TATC-box, and MeJA response elements CGTCA and TGACG motifs in the promotors of some CsTCPs (Figure 4 and Table S4).

Additionally, we identified plenty of cis-acting elements related to light response in these promotors, including G-Box, GAG-motif, I-box, AE-box, TCT-box, GATA-box, ATC-motif and GT1-motif. We also identified a large amount of defense and stress response elements, including W-box, Box-s, MBS, Myc, Myb and TC-rich (Figure 4 and Table S4).

\subsection{Expression Profiles of Putative Cucumber TCP Genes in Different Tissues}

We analyzed the expression of the 27 putative cucumber TCP genes (CsTCP1-CsTCP27) in different cucumber organs, including root, stem, leaf, cotyledon, tendril, and flower (petal, stamen, pistil, carpel, pericarp and trichome) by qRT-PCR (Figure 5, Table S5). The results showed that the CsTCPs presented distinct expression profiles in different organs, suggesting functional divergence of CsTCPS for plant development. The expression levels including CsTCP22 and CsTCP23 were consistently high in every organ. In addition, there were seven genes which have high expression level in most organs, including CsTCP4, CsTCP13, CsTCP15, CsTCP16, CsTCP18, CsTCP19 and CsTCP27. The expression level of these genes was even much higher than CsTCP22 and CsTCP23 in specific organs. In contrast, CsTCP7, CsTCP17, CsTCP20, CsTCP21 and CsTCP24 were not detected in most organs. Similarly, CsTCP3, CsTCP6, CsTCP7 and CsTCP8 were also expressed at a very low level in every organ. Interestingly, several low-expressed genes were found to express in specific tissue as follows: CsTCP10 had the strongest expression in carpel, and CsTCP21 showed specific expression had the strongest expression in tendril. These genes may play a role in the development of the corresponding phenotype. In addition, we found that CsTCPs had low expression level in root. This implied that CsTCPs mainly involved in the development of other organs rather than root in cucumber. 


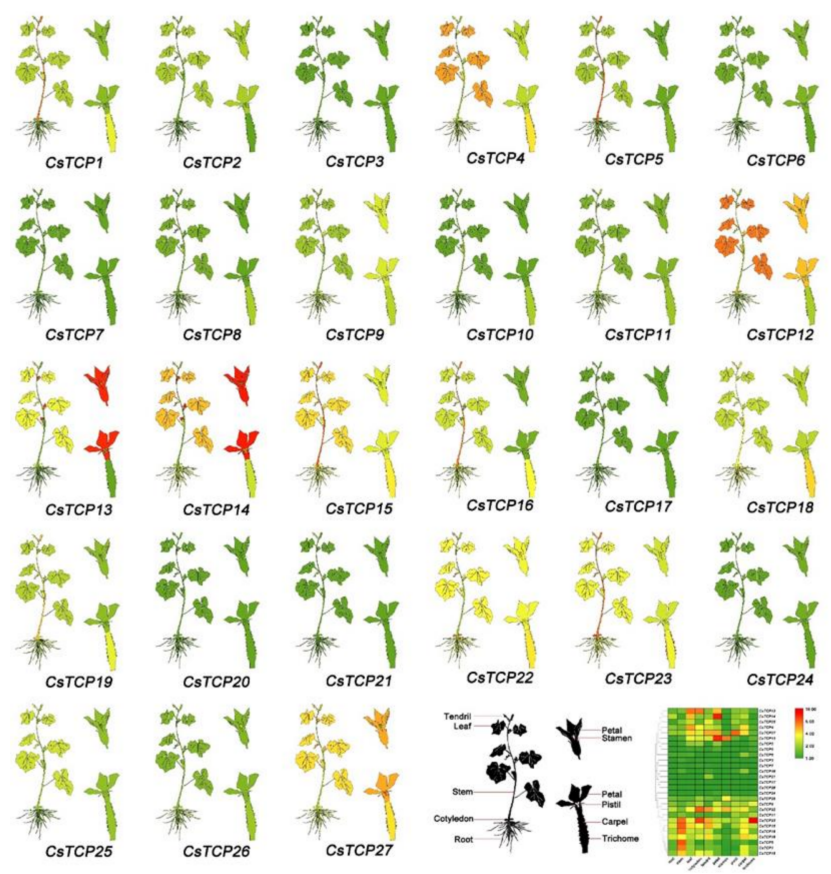

Figure 5. The differential expression of CsTCPs in different organs by qRT-PCR. The mean expression values were visualized by Tbtools; Red represents high expression level and green represent low expression level. The relative expression values and standard errors is provided in Table S5.

\subsection{Expression Analysis of CsTCPs during Flower Bud Development}

To elucidate their roles in flower development, the qRT-PCR were conducted to confirm the relative expression levels of 27 CsTCPs at different stages of flower bud development (Figure 6). The whole floral development process was divided into 12 stages (s1-s12) The development of female and male flowers were not morphologically distinguishable at s1-s5 (floral bud side: $0.1-0.55 \mathrm{~mm}$ ). The carpel primordia had just initiated in the female flower at $\mathrm{s} 6$ (floral bud side: $0.65 \mathrm{~mm}$ ), which is the key stage for morphologic divergence from normal to inappropriate organs [41]. Most CsTCPs were detected during the flower bud differentiation in cucumber line 9930 except CsTCP17 and CsTCP24, suggesting that most of them may regulate the flower development.

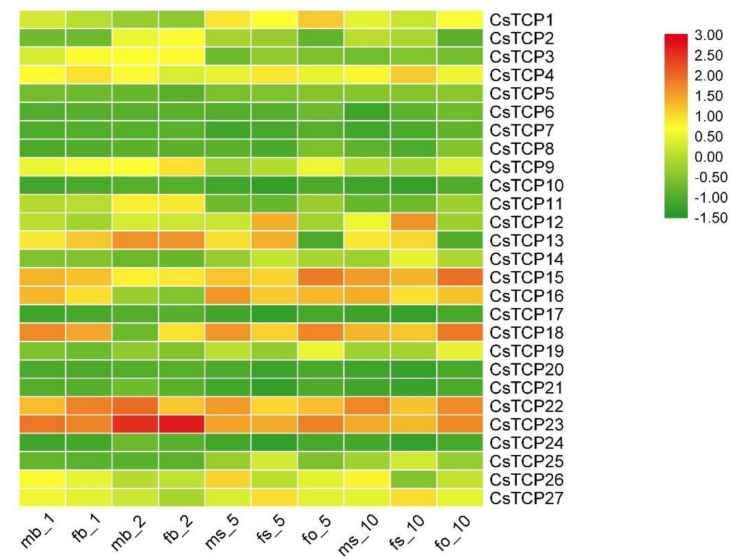

Figure 6. Expression analysis of CsTCPs in the development of female and male flower buds. The following abbreviations are used: mb_1, male bud $1 \mathrm{~mm}$; fb-1, female bud $1 \mathrm{~mm}$; fb_2, female bud $2 \mathrm{~mm}$; ms_5, male stamen $5 \mathrm{~mm}$; fs_5, female stigma $5 \mathrm{~mm}$; fo_5, female ovary $5 \mathrm{~mm}$; ms_10, male stamen $10 \mathrm{~mm}$; fs_10, female stigma $10 \mathrm{~mm}$; fo_10, female ovary $10 \mathrm{~mm}$. Genes highly expressed in organs are colored red, and genes lower expressed in organs are colored green. 
When the length of floral buds was $1 \mathrm{~mm}$, there is no difference in the expression among CsTCPS between female and male flower buds. However, CsTCP4, CsTCP12, CsTCP13, CsTCP14, CsTCP25, and CsTCP27, all belong to the CIN subclass, were expressed highly in stigma in the process of female bud differentiation (female flower bud side: 2-10 $\mathrm{mm}$ ) (Figure 6). Then, the expression of these genes increased with the development of the stigma, implying that these genes were involved in development of female floral organs. Besides, CsTCP15, CsTCP16, CsTCP18, CsTCP22 and CsTCP23 were always highly expressed in the early stage of female and male flower bud development. We could not detect the expression of CsTCP10, CsTCP17, CsTCP20, CsTCP21 and CsTCP24 in female and male buds with the length of floral organs increasing, implying that these genes should not be involved in the development of floral organs.

\subsection{Expression Analysis of CsTCPs Under Hormone Treatments and Environmental Stresses}

To demonstrate whether CSTCPs are involved in the pathway of hormone, we analyzed the expression of CsTCPs after GA and ethylene treatment. As shown in Figure 7, CsTCP3, CsTCP9, CsTCP19 were up-regulated after $12 \mathrm{~h}$ of GA treatment (Figure 7a). The expression level of CsTCP3 and CsTCP9 increased, while expression level of CSTCP19 decreased slightly after $24 \mathrm{~h}$ of GA treatment (Figure 7b). However, CsTCP25 was down-regulated and always maintained at a low level until $24 \mathrm{~h}$ (Figure 7a,b). Half a month after GA treatment, we found that the lateral organs, including male, female flower buds and lateral branches, were inhibited (Figure 7d,e and Figure S3). Previous studies showed that CSTCP3 inhibited axillary bud outgrowth [29]. We considered that up-regulated expression of CsTCP3 induced by GA treatment should suppress the lateral branch development in cucumber. After 12-24 h of ethylene treatment, the expression of most CsTCPS were not changed significantly, except CsTCP1. The expression of CSTCP1 was down-regulated and maintained at a low level (Figure 7c). Half a month after ethylene treatment, the number of lateral branches was increasing and the lateral branches were growing at the low number of nodes of plants (Figure 7e,f and Figure S3). The above results indicated that these CsTCPs may participate in the development of lateral organs in response to GA and ethylene in cucumber.
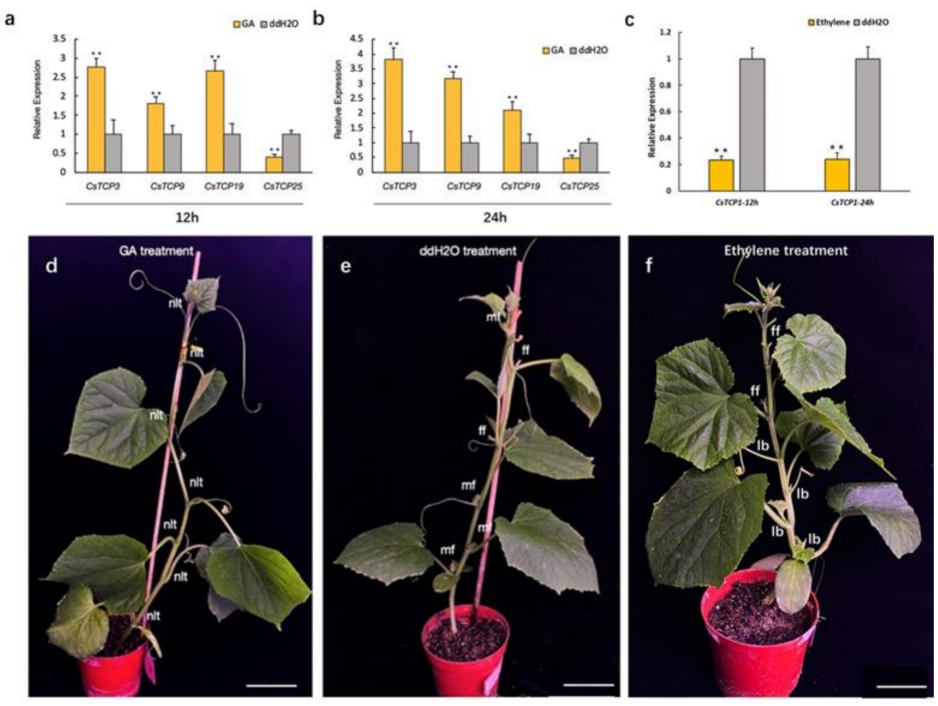

Figure 7. The Morphology and CsTCPs expression levels of cucumber plants under gibberellin (GA) and ethylene treatment. (a,b) Expression analysis of CsTCPs at $12 \mathrm{~h}(\mathbf{a})$ and $24 \mathrm{~h} \mathrm{(b)}$ after GA treatment, respectively. (c) Expression analysis of CsTCP1 at $12 \mathrm{~h}$ and $24 \mathrm{~h}$ after ethylene treatment. The $y$ axis is the scale of the relative transcript abundance level. Error bars represent the standard deviations from three biological replicates. ${ }^{* *}$ stands for significant difference $(p<0.01)$. (d-f) Morphology of plants after GA treatment (d), $\mathrm{ddH}_{2} \mathrm{O}$ treatment (e), and ethylene treatment (f). nlt, non-lateral tissues; mf, male flower; ff, female flower; $\mathrm{lb}$, lateral branch. 
To understand the functions of CsTCPs in the stress-related environmental adaptation, we analyzed the expression of CsTCPs and the phenotype of cucumber seedings treated with temperature and photoperiod. As shown in Figure 8, after different temperature and photoperiod treatments, the trend of the expression of CsTCP6 and CsTCP8 were similar. Compared with the normal condition, the expression of CSTCP6 and CsTCP8 decreased under LL and LS condition However, the CSTCP7 was up-regulated under LL and LS condition (Figure 8a). Additionally, CsTCP3 was induced significantly under LS condition, and was up-regulated slightly under LL and HS condition (Figure 8a). Compared with the normal condition, the number of lateral branches of the cucumber was significantly reduced, and the ratio of petiole of plant was also decreased under LL and LS condition (Figure 8b,c). These results indicated that CsTCPs may participate in the development of lateral organs in response to temperature and photoperiod in cucumber.
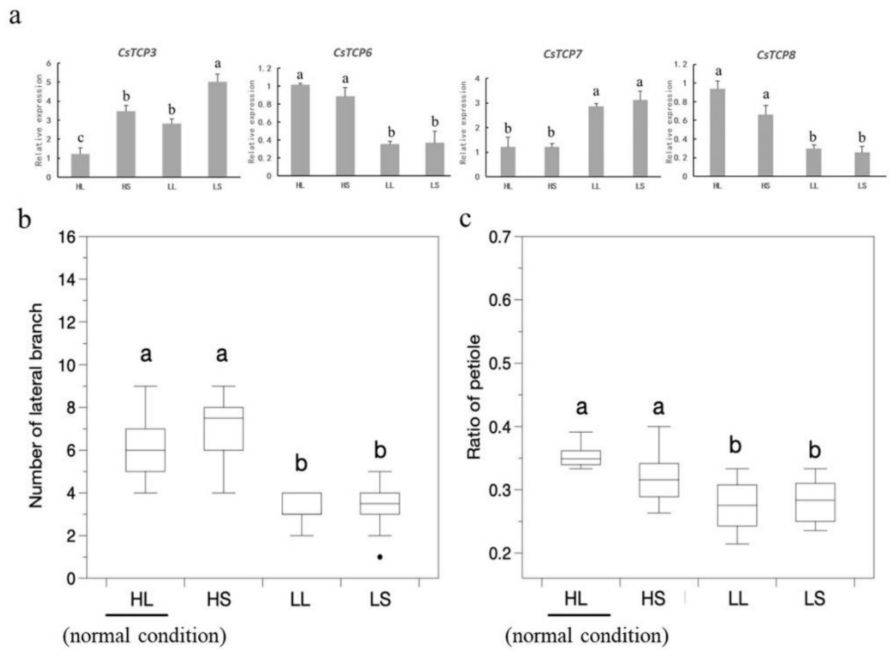

Figure 8. Phenotypes and CsTCPs expression levels of cucumber plants under different temperature and photoperiod treatments. (a) Expression analysis of four CsTCPs under different temperature and photoperiod treatment. (b) Comparison of the number of lateral branch and (c) Ratio of the petiole of cucumber under HL, HS, LL and LS condition. HL, high temperature and long day, as the normal condition; HS, high temperature and short day; LL, low temperature and long day; LS, low temperature and short day. Error bars represent the standard deviations from three biological replicates. Different letters indicate significant difference.

\section{Discussion}

Plant-specific transcription factors TCPs play various roles in multiple aspects of plant growth and development. In different species, the general organization of TCP family is conserved, and most of them are divided into two classes [9,11,42]. In cucumber genome, we identified 27 putative CsTCP genes, more than the previous study that identified 22 CsTCPs [30]. Phylogenetic analysis showed the 27 CsTCPs were divided into two classes, Class I and Class II. This result is consistent with the previous classification [6]. By analyzing the distribution of motifs, we found Motifs 4 and 5 are observed in most Class I members, and the great majority of members in the class I included Motif 2. Thus, the CsTCPs in the same class had similar motif composition. Similarly, miR319 binding sites analysis of CsTCPS had similar results. In cucumber, we identified four putative miR319-targeted TCP genes including CsTCP12, CsTCP14, CsTCP25 and CsTCP27, all of which belonged to CIN subclass. In general, genes in the same class shared the similar gene structure and motif distribution. However, the gene structure of CsTCPs in the same class had no obvious regularity. The reason may be that most putative CsTCP genes structure was relatively simple.

Most of CsTCPs in Class I had high expression level in every organ, except CsTCP24. CsTCP1, CsTCP5 and CsTCP19 were closely associated with Arabidopsis AtTCP14 (At3G47620) and AtTCP15 (At1G69690) (Figure S2). CsTCP1, CsTCP5 and CsTCP19 had the strongest expression in the stem. 
The AtTCP14 and AtTCP15 were involved in regulating the internode elongation and trichome branching in Arabidopsis [43,44]. CsTCP4 and CsTCP27 were highly expressed in carpel and pistil, respectively, and both maintained high expression levels in stigma during the female flower development. AtTCP2, an Arabidopsis ortholog of CsTCP4 and CsTCP27 was most strongly expressed in flowers, but not other organs [9]. These results suggest these genes may have a similar function in different species.

In CYC/TB1 subclass, most members were expressed at a very low level in all organs, especially CsTCP3, CsTCP7, CsTCP17 and CsTCP20 were not detected in almost organs. In addition, we found that some genes in CYC/TB1 subclass were expressed in specific organs. CsTCP10 showed specific expression in carpel, CsTCP21 had the specific expression in tendril. However, the Arabidopsis homolog of CsTCP10 and CsTCP21 is AtTCP1 (At1G67260), whose function is to regulate the elongation of leaves [45]. The CsTCPs presented distinct expression profiles in various organs, suggesting their functional divergences during cucumber development.

Previous studies have shown that TCP genes play a key role in flower development. PeCIN8 is involved in the regulation of ovule development in Phalaenopsis equestris [12]. In Prunus mume, the CIN type genes were highly expressed in ovule development stages and pistil initiation [39]. In this study, CsTCP12, CsTCP13, CsTCP14 and CsTCP25 all belong to the CIN subclass, which were also expressed highly in stigma, and their expressions increased with the development of the stigma. The result implied these CIN type genes play an important role in development of female floral organs in cucumber. The Class I type genes, CsTCP15, CsTCP16, CsTCP18, CsTCP22 and CsTCP23 were always highly expressed in the male and female flower bud development. Class I type gene in Chrysanthemum morifolium, CmTCP14 suppresses organ size and prolong flowering time [46]. In Arabidopsis, AtTCP11 (At2G37000) and AtTCP16 (At3G45150) participate in early pollen development [47,48]. These results suggested that CIN type and Class I type CsTCPs may be involved in the flower development of cucumber.

GA and ethylene are the key endogenous regulator of plant development and growth $[49,50]$. In our study, we found that the growth of lateral organs was inhibited after GA treatment, and the lateral branches grew at the low nodes of plants after ethylene treatment. The cis-acting elements analysis of 27 CsTCPS showed that several GA-related cis-elements and ERE elements in their promotors were identified. The expression of some CsTCPs were significantly changed after GA treatment, including CsTCP3 and CsTCP19. However, although the promotors of CsTCP9 and CsTCP25 contain no GA-related cis-elements, the expression of CsTCP9 and CsTCP25 was up-regulated and down-regulated after GA treatment, respectively. These results suggested that GA affects the development of lateral organs by directly or indirectly regulating the expression of CsTCPs. The pea PsBRC1 acted as a typical branch number regulator, involving in GA signal [20]. Although many CsTCPS contain ERE elements, ethylene treatment did not affect the expression of these genes. While CsTCP1 contains no ERE elements, its expression was significant decreased and maintained at low level after ethylene treatment. These results indicated that CsTCP1 should regulate the lateral branch development by participating in ethylene signal pathway. Motif 1 was presented in almost CsTCPs except CsTCP 1. CsTCP1 lacking Motif 1 may have acquired new functions during evolution in cucumber.

Environment conditions show pronounced impacts on the shoot branching habit of plant [51,52]. In this study, we found that the number of lateral branches was increasing under shorter photoperiod and low temperature, and four CsTCPs were induced by the environment changes. It demonstrated that the four putative CsTCP genes may participate in the lateral branch development by responding to temperature signals. The CsTCP3 was induced by the low temperature and short photoperiod, and it was also induced by GA treatment. Previous studies suggested that CsTCP3 suppresses the lateral bud growth by controlling the accumulation of auxin in cucumber axillary buds [29]. We found that both of CsTCP3 (Csa1G020890.1) and CsTCP7 (Csa1G042180.1) were closely associated with Arabidopsis AtTCP18 (BRC1). AtTCP18 plays a vital role in the control of shoot branching [39]. Hence, we speculated that CSTCP3 may regulate lateral branching development by participating in multiple signaling pathways. 
Supplementary Materials: The following are available online at http://www.mdpi.com/2073-4425/11/11/1379/s1, Table S1: Primers for qRT-PCR in this study, Table S2: The basic information of 27 putative TCP genes, Table S3: Collinear relationships of TCP family members in the cucumber and between the melon and Arabidopsis, Table S4: Cis-acting elements on promotors of CsTCPs, Table S5: Expression levels of cucumber TCP genes in different tissues, Figure S1: Physical locations of putative TCP genes on cucumber chromosomes. Scale bar on the left represents the length of the chromosome(bp), Figure S2: Phylogenetic tree of TCPs in Arabidopsis and cucumber. The phylogenetic tree was constructed based on TCPs sequences from cucumber (27 proteins, marked with black dot) and Arabidopsis (24 proteins). A specific color indicates each of the three classes, Figure S3: Comparison the number of lateral tissues of cucumber under GA and ethylene treatments. Error bars represent the standard deviations from three biological replicates $\left(^{* *}<0.01\right.$, Student's $t$ test).

Author Contributions: Conceptualization, G.W.; Data curation, Y.C., H.D., L.Z., K.Z.; Resources, H.H., J.P. and R.C.; Software, Y.C.; Validation, G.W.; Writing-original draft, H.W.; Writing-review \& editing, H.W. and G.W. All authors have read and agreed to the published version of the manuscript.

Funding: This research was funded by National Natural Science Foundation of China (31672173).

Conflicts of Interest: The authors declare no conflict of interest and the sponsors had no role in the design, execution, interpretation, or writing of the study.

\section{References}

1. Meshi, T.; Iwabuchi, M. Plant Transcription Factors. Plant Cell Physiol. 1995, 36, 1405-1420. [PubMed]

2. Doebley, J.; Stec, A.; Hubbard, L. The evolution of apical dominance in maize. Nature 1997, 386, 485-488. [CrossRef] [PubMed]

3. Luo, D.; Carpenter, R.; Vincent, C.; Copsey, L.; Coen, E. Origin of floral asymmetry in Antirrhinum. Nature 1996, 383, 794-799. [CrossRef] [PubMed]

4. Kosugi, S.; Ohashi, Y. PCF1 and PCF2 specifically bind to cis elements in the rice proliferating cell nuclear antigen gene. Plant Cell 1997, 9, 1607-1619. [PubMed]

5. Cubas, P.; Lauter, N.; Doebley, J.; Coen, E. The TCP domain: A motif found in proteins regulating plant growth and development. Plant J. 1999, 18, 215-222. [CrossRef] [PubMed]

6. Martin-Trillo, M.; Cubas, P. TCP genes: A family snapshot ten years later. Trends Plant Sci. 2010, 15, 31-39. [CrossRef]

7. Howarth, D.; Donoghue, M. Phylogenetic analysis of the "ECE" (CYC/TB1) clade reveals duplications predating the core eudicots. Proc. Natl. Acad. Sci. USA 2006, 103, 9101-9106. [CrossRef]

8. Navaud, O.; Dabos, P.; Carnus, E.; Tremousaygue, D.; Hervé, C. TCP Transcription Factors Predate the Emergence of Land Plants. J. Mol. Evol. 2007, 65, 23-33. [CrossRef]

9. Yao, X.; Ma, H.; Wang, J.; Zhang, D. Genome-Wide Comparative Analysis and Expression Pattern of TCP Gene Families in Arabidopsis thaliana and Oryza sativa. J. Integr. Plant Biol. 2007, 49, 885-897. [CrossRef]

10. Liu, M.M.; Wang, M.M.; Yang, J.; Wen, J.; Guo, P.C.; Wu, Y.W.; Ke, Y.Z.; Li, P.F.; Li, J.N.; Du, H. Evolutionary and Comparative Expression Analyses of TCP Transcription Factor Gene Family in Land Plants. Int. J. Mol. Sci. 2019, 20, 3591. [CrossRef]

11. Ma, J.; Wang, Q.; Sun, R.; Xie, F.; Jones, D.C.; Zhang, B. Genome-wide identification and expression analysis of TCP transcription factors in Gossypium raimondii. Sci. Rep. 2014, 4, 6645. [CrossRef] [PubMed]

12. Lin, Y.F.; Chen, Y.Y.; Hsiao, Y.Y.; Shen, C.Y.; Hsu, J.L.; Yeh, C.M.; Mitsuda, N.; Ohme-Takagi, M.; Liu, Z.J.; Tsai, W.C. Genome-wide identification and characterization of TCP genes involved in ovule development of Phalaenopsis equestris. J. Exp. Bot. 2016, 67, 5051-5066. [CrossRef] [PubMed]

13. Bresso, E.G.; Chorostecki, U.; Rodriguez, R.E.; Palatnik, J.F.; Schommer, C. Spatial Control of Gene Expression by miR319-Regulated TCP Transcription Factors in Leaf Development. Plant Physiol. 2018, 176, 1694-1708. [CrossRef] [PubMed]

14. Wang, X.; Xu, X.; Mo, X.; Zhong, L.; Zhang, J.; Mo, B.; Kuai, B. Overexpression of TCP8 delays Arabidopsis flowering through a FLOWERING LOCUS C-dependent pathway. BMC Plant Biol. 2019, 19, 534. [CrossRef] [PubMed]

15. Koyama, T.; Mitsuda, N.; Seki, M.; Shinozaki, K.; Ohme-Takagi, M. TCP transcription factors regulate the activities of ASYMMETRIC LEAVES1 and miR164, as well as the auxin response, during differentiation of leaves in Arabidopsis. Plant Cell 2010, 22, 3574-3588. [CrossRef] [PubMed] 
16. Wang, M.Y.; Zhao, P.M.; Cheng, H.Q.; Han, L.B.; Wu, X.M.; Gao, P.; Wang, H.Y.; Yang, C.L.; Zhong, N.Q.; Zuo, J.R.; et al. The cotton transcription factor TCP14 functions in auxin-mediated epidermal cell differentiation and elongation. Plant Physiol. 2013, 162, 1669-1680. [CrossRef] [PubMed]

17. Ju, Y.; Guo, L.; Cai, Q.; Ma, F.; Zhu, Q.Y.; Zhang, Q.; Sodmergen. Arabidopsis JINGUBANG Is a Negative Regulator of Pollen Germination That Prevents Pollination in Moist Environments. Plant Cell 2016, 28, 2131-2146. [CrossRef]

18. Zhang, W.; Cochet, F.; Ponnaiah, M.; Lebreton, S.; Matheron, L.; Pionneau, C.; Boudsocq, M.; Resentini, F.; Huguet, S.; Blázquez, M.Á.; et al. The MPK8-TCP14 pathway promotes seed germination in Arabidopsis. Plant J. 2019, 100, 677-692. [CrossRef]

19. Li, D.; Zhang, H.; Mou, M.; Chen, Y.; Xiang, S.; Chen, L.; Yu, D. Arabidopsis Class II TCP Transcription Factors Integrate with the FT-FD Module to Control Flowering. Plant Physiol. 2019, 181, 97-111. [CrossRef]

20. Braun, N.; de Saint Germain, A.; Pillot, J.P.; Boutet-Mercey, S.; Dalmais, M.; Antoniadi, I.; Li, X.; Maia-Grondard, A.; Le Signor, C.; Bouteiller, N.; et al. The pea TCP transcription factor PsBRC1 acts downstream of Strigolactones to control shoot branching. Plant Physiol. 2012, 158, 225-238. [CrossRef]

21. Gastaldi, V.; Lucero, L.E.; Ferrero, L.V.; Ariel, F.D.; Gonzalez, D.H. Class-I TCP Transcription Factors Activate the SAUR63 Gene Subfamily in Gibberellin-Dependent Stamen Filament Elongation. Plant Physiol. 2020, 182, 2096-2110. [CrossRef] [PubMed]

22. Gao, Y.; Zhang, D.; Li, J. TCP1 Modulates DWF4 Expression via Directly Interacting with the GGNCCC Motifs in the Promoter Region of DWF4 in Arabidopsis thaliana. J. Genet. Genom. 2015, 42, 383-392. [CrossRef] [PubMed]

23. Van Es, S.W.; Silveira, S.R.; Rocha, D.I.; Bimbo, A.; Martinelli, A.P.; Dornelas, M.C.; Angenent, G.C.; Immink, R.G.H. Novel functions of the Arabidopsis transcription factor TCP5 in petal development and ethylene biosynthesis. Plant J. 2018, 94, 867-879. [CrossRef] [PubMed]

24. Wu, J.F.; Tsai, H.L.; Joanito, I.; Wu, Y.C.; Chang, C.W.; Li, Y.H.; Wang, Y.; Hong, J.C.; Chu, J.W.; Hsu, C.P.; et al. LWD-TCP complex activates the morning gene CCA1 in Arabidopsis. Nat. Commun. 2016, 7, 13181. [CrossRef]

25. Mohammadi, A.; Omid, M. Economical analysis and relation between energy inputs and yield of greenhouse cucumber production in Iran. Appl. Energy 2010, 87, 191-196. [CrossRef]

26. Malepszy, S.; Niemirowicz-Szczytt, K. Sex determination in cucumber (Cucumis sativus) as a model system for molecular biology. Plant Sci. 1991, 80, 39-47. [CrossRef]

27. Wang, S.; Yang, X.; Xu, M.; Lin, X.; Lin, T.; Qi, J.; Shao, G. A Rare SNP Identified a TCP Transcription Factor Essential for Tendril Development in Cucumber. Mol. Plant 2015, 8, 1795-1808. [CrossRef]

28. Yang, X.; Yan, J.; Zhang, Z.; Lin, T.; Xin, T.; Wang, B.; Wang, S.; Zhao, J.; Zhang, Z.; Lucas, W.J.; et al. Regulation of plant architecture by a new histone acetyltransferase targeting gene bodies. Nat. Plants 2020,6, 809-822. [CrossRef]

29. Shen, J.; Zhang, Y.; Ge, D.; Wang, Z.; Song, W.; Gu, R.; Che, G.; Cheng, Z.; Liu, R.; Zhang, X. CsBRC1 inhibits axillary bud outgrowth by directly repressing the auxin efflux carrierCsPIN3 in cucumber. Proc. Natl. Acad. Sci. USA 2019, 116, 17105-17114. [CrossRef]

30. Yuan, W.; Gu, Z.; Chu, W.; Ye, L.; Yang, G. Identification and structure analysis of TCP transcription factors in Cucumber. Mol. Plant Breed. 2014, 12, 287-295. (In Chinese)

31. Chen, C.; Xia, R.; Chen, H.; He, Y. TBtools, a Toolkit for Biologists integrating various HTS-data handling tools with a user-friendly interface. bioRxiv 2018, 289660.

32. Letunic, I.; Bork, P. 20 years of the SMART protein domain annotation resource. Nucleic Acids Res. 2017, 46, D493-D496. [CrossRef] [PubMed]

33. El-Gebali, S.; Mistry, J.; Bateman, A.; Eddy, S.R.; Luciani, A.; Potter, S.C.; Qureshi, M.; Richardson, L.J.; Salazar, G.A.; Smart, A.; et al. The Pfam protein families database in 2019. Nucleic Acids Res. 2018, 47, D427-D432. [CrossRef] [PubMed]

34. Subramanian, B.; Gao, S.; Lercher, M.J.; Hu, S.; Chen, W.-H. Evolview v3: A webserver for visualization, annotation, and management of phylogenetic trees. Nucleic Acids Res. 2019, 47, W270-W275. [CrossRef] [PubMed]

35. Bailey, T.L.; Boden, M.; Buske, F.A.; Frith, M.; Grant, C.E.; Clementi, L.; Ren, J.; Li, W.W.; Noble, W.S. MEME Suite: Tools for motif discovery and searching. Nucleic Acids Res. 2009, 37, W202-W208. [CrossRef] 
36. Wang, Y.; Tang, H.; Debarry, J.D.; Tan, X.; Li, J.; Wang, X.; Lee, T.-H.; Jin, H.; Marler, B.; Guo, H.; et al. MCScanX: A toolkit for detection and evolutionary analysis of gene synteny and collinearity. Nucleic Acids Res. 2012, 40, e49. [CrossRef]

37. Livak, K.J.; Schmittgen, T.D. Analysis of relative gene expression data using real-time quantitative PCR and the 2(-Delta Delta C(T)) Method. Methods 2001, 25, 402-408. [CrossRef]

38. Ma, X.; Ma, J.; Fan, D.; Li, C.; Jiang, Y.; Luo, K. Genome-wide Identification of TCP Family Transcription Factors from Populus euphratica and Their Involvement in Leaf Shape Regulation. Sci. Rep. 2016, 6, 32795. [CrossRef]

39. Zhou, Y.; Xu, Z.; Zhao, K.; Yang, W.; Cheng, T.; Wang, J.; Zhang, Q. Genome-Wide Identification, Characterization and Expression Analysis of the TCP Gene Family in Prunus mume. Front. Plant Sci. 2016, 7, 1301. [CrossRef]

40. Huang, S.; Li, R.; Zhang, Z.; Li, L.; Gu, X.; Fan, W.; Lucas, W.J.; Wang, X.; Xie, B.; Ni, P.; et al. The genome of the cucumber, Cucumis sativus L. Nat. Genet. 2009, 41, 1275-1281. [CrossRef]

41. Bai, S.L.; Peng, Y.B.; Cui, J.X.; Gu, H.T.; Xu, L.Y.; Li, Y.Q.; Xu, Z.H.; Bai, S.N. Developmental analyses reveal early arrests of the spore-bearing parts of reproductive organs in unisexual flowers of cucumber (Cucumis sativus L.). Planta 2004, 220, 230-240. [CrossRef] [PubMed]

42. Chen, L.; Chen, Y.Q.; Ding, A.M.; Chen, H.; Xia, F.; Wang, W.F.; Sun, Y.H. Genome-wide analysis of TCP family in tobacco. Genet. Mol. Res. 2016, 15, gmr.15027728. [CrossRef]

43. Murray, J.; Viola, I.L.; Gonzalez, D.H.; Alem, A.L.; Ariel, F.D.; Arce, A.L.; Camoirano, A. Class I TCP transcription factors regulate trichome branching and cuticle development in Arabidopsis. J. Exp. Bot. 2020, 71, 5438-5453.

44. Kieffer, M.; Master, V.; Waites, R.; Davies, B. TCP14 and TCP15 affect internode length and leaf shape in Arabidopsis. Plant J. 2011, 68, 147-158. [CrossRef] [PubMed]

45. Koyama, T.; Sato, F.; Ohme-Takagi, M. A role of TCP1 in the longitudinal elongation of leaves in Arabidopsis. Biosci. Biotechnol. Biochem. 2010, 74, 2145-2147. [CrossRef] [PubMed]

46. Zhang, T.; Qu, Y.; Wang, H.; Wang, J.; Song, A.; Hu, Y.; Chen, S.; Jiang, J.; Chen, F. The heterologous expression of a chrysanthemum TCP-P transcription factor CmTCP14 suppresses organ size and delays senescence in Arabidopsis thaliana. Plant Physiol. Biochem. 2017, 115, 239-248. [CrossRef]

47. Takeda, T.; Amano, K.; Ohto, M.-a.; Nakamura, K.; Sato, S.; Kato, T.; Tabata, S.; Ueguchi, C. RNA interference of the Arabidopsis putative transcription factor TCP16 gene results in abortion of early pollen development. Plant Mol. Biol. 2006, 61, 165-177. [CrossRef]

48. Viola, I.L.; Manassero, N.G.U.; Ripoll, R.; Gonzalez, D.H. The Arabidopsis class I TCP transcription factor AtTCP11 is a developmental regulator with distinct DNA-binding properties due to the presence of a threonine residue at position 15 of the TCP domain. Biochem. J. 2011, 435, 143-155. [CrossRef]

49. Katyayini, N.U.; Rinne, P.L.; Tarkowská, D.; Strnad, M.; van der Schoot, C. Dual Role of Gibberellin in Perennial Shoot Branching: Inhibition and Activation. Front. Plant Sci. 2020, 11, 736. [CrossRef]

50. Wang, D.H.; Li, F.; Duan, Q.H.; Han, T.; Xu, Z.H.; Bai, S.N. Ethylene perception is involved in female cucumber flower development. Plant J. 2010, 61, 862-872. [CrossRef]

51. Drummond, R.S.; Janssen, B.J.; Luo, Z.; Oplaat, C.; Ledger, S.E.; Wohlers, M.W.; Snowden, K.C. Environmental control of branching in petunia. Plant Physiol. 2015, 168, 735-751. [CrossRef] [PubMed]

52. Rameau, C.; Bertheloot, J.; Leduc, N.; Andrieu, B.; Foucher, F.; Sakr, S. Multiple pathways regulate shoot branching. Front. Plant Sci. 2014, 5, 741. [CrossRef] [PubMed]

Publisher's Note: MDPI stays neutral with regard to jurisdictional claims in published maps and institutional affiliations.

(C) 2020 by the authors. Licensee MDPI, Basel, Switzerland. This article is an open access article distributed under the terms and conditions of the Creative Commons Attribution (CC BY) license (http://creativecommons.org/licenses/by/4.0/). 